\title{
A Quadratic Anthropocentric Perspective on Feedback - Using Proxemics as a Framework
}

\author{
Diana Saplacan \\ University of Oslo, Department of Informatics \\ Postbox 1080 Blindern, 0316 Oslo, Norway \\ diana.saplacan@uio.no
}

\author{
Jo Herstad \\ University of Oslo, Department of Informatics \\ Postbox 1080 Blindern, 0316 Oslo, Norway \\ jo.herstad@uio.no
}

\begin{abstract}
This paper is a conceptual paper discussing feedback, from a quadratic anthropocentric perspective where the feedbacks' source, e.g. the emitters, may be humans, non-digital technology, still- and inmotion technologies. Here, both the source- and the receiver of feedback are co-present, i.e. share a physical space. Still technologies mean here non-autonomous digital technologies, i.e. technologies that do not move by themselves, whereas in-motion technologies are defined here as semiautonomous digital technologies. The aim of the paper is to gestate the concept of feedback, and to inform the Multimodal-Elderly-Care-System (MECS) project, on design of robots for the stay-at-home elderly. The purpose of the paper is to advance the discussions within the human-computer interaction $(\mathrm{HCl})$ field, around the concept of feedback frameworked by spatiality - more exactly proxemics.
\end{abstract}

Feedback, humans, still technologies, in-motion technologies, co-presence, proxemics, elderly

\section{INTRODUCTION}

In the interaction with other humans, non-digital technologies, and digital technologies, one often uses the term feedback for describing a particular type of interaction. Usually, one has a preunderstanding of the term, and it often encompasses some kind of response to a previously-performed action. The term is used across fields and disciplines, from cybernetics and control theory, to human computer interaction $(\mathrm{HCl})$, from pedagogics and education, to various organizational studies, but also in everyday communication. The use of the term often indicates the invocation of an output to an input, a response, reply, answer, comment, reaction, or evaluation. This paper starts with presenting the aim and purpose, including the case of an ongoing research project, Multimodal Elderly Care System (MECS) ${ }^{i}$, the research question, and a brief on proxemics. Thereafter, relevant literature is presented as background for this contribution. The paper continues then with a quadratic anthropocentric perspective on how the feedback may be perceived by humans, e.g. here a receiver of feedback, in relation to its source, e.g. the emitter, such as: other humans, non-digital technology, and digital still- and in motion technologies. Finally, this paper concludes with some final remarks and suggestions for future work.

\section{AIM AND PURPOSE}

The aim of this paper is two-fold: to inform the research project Multimodal Elderly Care System (MECS), and to gestate the concept of feedback with regard to spatiality, i.e. proxemics and co-presence.

\subsection{Multimodal Elderly Care System (MECS) Project}

MECS is an ongoing project at University of Oslo, funded by the Research Council of Norway, intended to investigate in-motion technologies, e.g. robots, for the stay-at-home elderly. Amongst the project collaborators is Kampen Omsorg Pluss, an organization providing accommodation for the independent stay-at-home elderly. The paper presents some of the first findings during the gathering of system requirements while working with participatory approaches.

\subsection{Purpose and research question}

The purpose of this paper is to: give insights on how feedback may be perceived by humans in the interaction between humans and other humans, non-digital technologies, and digital technologies, that may be still- or in motion technologies, and to advance the discussions between the relation of 
feedback and spatiality. Table 1 below shows these and their annotations used later in this paper.

Table 1: A quadratic anthropocentric perspective on feedback

\begin{tabular}{|l|c|c|}
\hline Perceiver & Emitter/Source & Annotation \\
\hline \multirow{4}{*}{ Human } & Human & $H-H$ \\
\cline { 2 - 3 } & Non-digital technology & $H-n T$ \\
\cline { 2 - 3 } & Still Technology & $H-s T$ \\
\cline { 2 - 3 } & In-Motion Technology & $H-m T$ \\
\hline
\end{tabular}

The question explored in this short contribution is:

What do we mean by feedback depending on the source that emits it, where both the receiver and emitter of feedback are co-present, i.e. share a physical space?

\subsection{On Proxemics}

Hall et al., (1968) defined proxemics as: "the study of people's use of their perceptual apparatus in different emotional states during different activities, in different relationships, settings, and contexts." Per Hall, (1966), and Hall et al., (1968), there are cultural pre-integrated patterns that structure the spatiality of communication between two actors: how close, or how far they are from each other. The authors identified here four types of such proximity: intimate, personal, social and public distance (see Hall, 1966, pp. 113-125). The type of distance between the two actors, the receiver and emitter, is strongly correlated with the relation between them, but also with their perceptions of the context. In this paper, the conceptualization of feedback is explored, with regard to phenomenon of copresence in proxemics.

\section{BACKGROUND}

According to Oxford English Dictionary (OED), the term feedback is based on the verb feed and the adverb back, and it was initially related to the action of: feeding, nourishing, give food to, sustaining, and fostering. Later, around the beginning of the 19th century, the term was used in relation to machines, and was defined as: "the return of a fraction of an output signal to the input of an earlier stage" and as "information about the results of a process". In the next subsection, we continue with its definition within $\mathrm{HCl}$ field.

\subsection{Relevant Literature}

Within cybernetics and control theory, feedback was coined as the process where several systems that influence each other (Åström and Murray, 2008), either by one of the systems being dominant, or each of the systems affecting each other (Ashby, 1957), usually involving a closed loop of causeeffect. In this paper, the phenomenon of feedback is being discussed with relation to $\mathrm{HCl}$. The two systems are here humans, and technologies. Pérez-Quiñones and Sibert (1996) argue that feedback is: "a necessary component of both human-human and human computer interaction". Specifically, from (ibid), according to Shneiderman (1986), in $\mathrm{HCl}$, feedback refers to a system's response to users' action. Norman, (2013) explains feedback as "some way of letting [the user knowing] that the system is working" on the user's request, being a way of "communicating the results of an action" (p. 22). Baecker, (1995), Lewis and Norman (1995) show that feedback can also be used for correction, as a remainder "to the deviation from the intention".

More recent studies focus mainly on feedback as a way to inform the user about the environmental impact or energy consumption, and as a multimodal form of interacting with-, or informing the user in affective computing. We give a brief on each of these.

First, there are studies on the aspect of ecology of feedback, so called eco-feedback, as shown by Barreto et al., (2013), Froehlich et al., (2010), Meurer et al., (2016), and Piccolo et al., (2016). These studies focus mainly on the sustainability and interaction design aspect of feedback. Here, the systems would sense the environment, and inform through eco-feedback about the energy consumption behavior. This can be up-to-date both for the domestic- or work environment, private or public spaces. This is also often related to ways of learning about one's own consumer behavior and habits, with the aim of internalizing that knowledge (Houwelingen et al., 1989, p. 99), and eventually changing it. Usually, the purpose of this type of feedback is to reduce the environmental impact by informing the user about the state of a system. Moreover, it requires longitudinal studies to track consumer behavior, and eventually predict future behavior (see Schwartz et al., 2015, 2013).

Second, feedback is also extensively discussed within the field of affective computing, which is defined as "computing that relates to, arises from or deliberately influences emotions" (Picard, 1995). Affective computing focuses on identifying, extracting and measuring "multimodal signals", i.e. emotions expressed through speech or facial recognition, in the interactions between humans and computers (Rukavina et al., 2016). The extracted information can later eventually be used as bio- or multimodal feedback. Bio-feedback is the process that "[enables] an individual to learn over time how to change his/her physiological activity for the purposes of improving health and performance" (Chittaro and Sioni, 2014, p. 663). Here, feedback is often expressed through multimodal medium: acoustic (ibid), visual, haptic, video etc. (Akshita et al., 2015; Kächele et al., 2014; Schels et al., 2014). 
Moreover, besides being an attribute of biofeedback, multimodality is also especially emphasized when developing systems for vulnerable groups, such as elderly (Biswas and Langdon, 2012; Rhiu et al., 2015; Sáenz-de-Urturi et al., 2015).

Finally, a lot of focus has been so far on ways of representing feedback. However, not much could be found on the relation between these representations of feedback and its spatiality: how does spatiality affect the way humans perceive feedback? We propose to open this discussion due to the increasing predicted number of household robots in homes that will follow in the up-coming yearsii.

\section{CONCEPTUALIZING FEEDBACK}

This section introduces the phenomena of feedback viewed through a phenomenological quadratic anthropocentric perspective, with the purpose of opening discussions towards the relation between feedback and co-presence in proxemics.

\subsection{Feedback: H-H}

Individuals often give feedback to each other, in formal or informal ways: at home, with friends and acquaintances, or in work situations. In informal situations, sometimes the term is used through its synonyms, such as: advice, opinion, view, judgement, idea, reflection, or consideration. Thus, when a human being asks for feedback from other human beings, they ask for a reflection of the others on something, an action, situation, or event, that has some type of relation to the inquirer. In this way, the individual is asking for nourishment or support from the other, in terms of thoughts. The inquirer relies on the other's instrument of thought, previous experiences and judgement, with the purpose of learning, and eventually calibrating his/her, or others' actions in the future. Specifically, in this subsection, it is elaborated on the phenomena of feedback between two humans, $\mathrm{H}-\mathrm{H}$, where both are present (co-present). This, of course, can vary, depending on the relation, the context and the setting. However, what it can be noticed here, is that the receiver, cannot control or predict the feedback received, this becoming a tacit dimension (see Polanyi, 1966). The feedback here can also be discussed in terms of explicit or implicit, direct or indirect feedback, including verbal, gestures and body language - but it will always manifest there and then, in the shared space, in some way. Is an un-manifested response, e.g. silence, stillness, still a feedback then?

\subsection{Feedback: H-nT}

A second type of feedback, is when one interacts in his/her daily lives with all kind of non-digital technologies. Such examples of non-digital technology may include: paper books, a pencil, a notebook. Feedback is considered here, the result of an action performed by the human. For example: let's say that the human, the receiver of feedback, the actant, performs the action of opening a book. In this sense, when the action has been performed, one could say that the actant gets a static instant visual feedback of the book being open. When the actant performs the action of writing in a notebook with a pencil, $s /$ he also get instant visual feedback, as s/he can see what has been written down. In this cases, the feedback is explicit. Both actions involve tactile feedback, as the actant needs to perform the actions through his/her own body. Underneath, the actant needs to have a relation, often close or direct with the things. In terms of proxemics, one can therefore talk about an intimate distance between the actant and the object of feedback (other things). S/he often does not ask for feedback, or is not aware of receiving this kind of feedback, unless there is a breakdown, the expected result is different, e.g. the pencil does not work, and eventually has to be corrected (Baecker, 1995; Lewis and Norman, 1995). When the result of the action performed is the expected one, the outcome could be considered as a positive feedback, whereas when it is not the expected one, it can be considered a negative one. Compared to feedback between $\mathrm{H}-\mathrm{H}$, the human, a receiver, is here in control of it there and then, and through the own body, as it has an intimate relation to the object of feedback.

\subsection{Feedback: H-sT}

We chose here to look at how feedback may be perceived by humans, here elderly, when the technologies are still, such as a smartphone, i.e. it does not move autonomously. This is exemplified here through the use of a smartphone.

Winograd and Flores, (1986) argue that in order to understand something, it requires a preunderstanding as a result of a previous experience. In the case of technologies, this implies that one has to be familiar with the technology in itself, or with an earlier version of it. For instance, the use of a smartphone becomes easier when one has already used a mobile phone earlier, or at least has used a phone. In that case, the technology feels more intuitive to the end-user (Turner, 2008). However, feedback in old and new phones may differ. The feedback here may be: auditive, haptic, visual, and even thermal, i.e. if we think about the heat that is emitted when it is used for too long, or if it is not properly charged. The feedback can be immediate when it is triggered by the user through the press of 
a button or keyboard. The user has control at some degree on the feedback received. When the phone rings, or when the user gets a notification, is less in control of the feedback received. The haptic feedback can be direct and tactile, through direct contact with the phone's surface, or kinesthetic, when the phone, for instance, vibrates. The visual feedback manifests usually through the screen. Here, the humans need to have here some kind of proximity to the phone in order to interact with it.

Proxemics can be discussed in terms of implicit and explicit feedback. Here, instead of talking about a receiver or transmitter of the feedback, one can talk about the user and the still technology. When the user and the still technology are co-present, it can be discussed, on one hand, about a close, intimate relationship, and, on the other hand, about a distant, personal relationship. In the former one, the user acts directly upon the phone, through tactile action, and expects an instant feedback: in the form of a visual feedback from the graphical user interface, auditory through sound notifications; kinesthetic through touch. In the latter one, the user is given feedback without necessarily asking for it: for instance when the user is getting a notification of a message, someone calls, the phone is signalizing through light or sound that it needs to be charged. In addition, one could also discuss the concept of extended proxemics, where the user is given feedback through the phone, while communicating with the external world, when for instance the user accesses an app or a web-browser. In this case, the smartphone becomes a medium for accessing information and communicating with the outside world. Here as well, it is matter of humans being in control of the feedback. On one hand, the user can act upon the smartphone and expect a specific result from the phone itself. In this case, one could say that the human is controlling the feedback. On the other hand, the user can act upon the extended proxemics, e.g. the outside world, feedback being explicitly requested. In this case, the human is not necessarily in control of the feedback, as s/he may or may not get a response from the outside world. However, when we refer to implicit feedback, the user may or may not notice it: for instance thermal feedback, or a small vibration. There is also the situation when the phone is in the silent mode, and although the user is notified about a coming message or phone call, the feedback becomes implicit, as it does not unfold through its evidenceness. But all in all, the takeaway idea is that the intimate co-presence between $\mathrm{H}$-sT facilitates an extended co-presence, to the outside world, such that the feedback is no longer limited to only the physical presence and intimate distance, as in $\mathrm{H}-\mathrm{H}$ and $\mathrm{H}-\mathrm{nT}$ explained in previous sections.

\subsection{Feedback: H-mT}

In this paper, in-motion technology, such as a robot, is defined as a digital still technology, but in addition it has the motion element. In this way, such a technology may include the same type of feedback as a still-technology, including the extended proxemics. In addition, the motion of the robot is identified as a type of feedback. In order to exemplify the feedback from in-motion technologies, feedback in robots for the stay-athome elderly was chosen. During the MECS prestudy phase, various types of robots, such as: AIBO ${ }^{i i i}, N^{2}{ }^{i v}$, and a Turtle Botv were brought in discussion and were part of the initial experiments. An immediate finding was that many of the elderly were not very familiar with the notion of a robot. However, vacuum cleaners and lawn mowers, for the use at home, were highly emphasized and seemed familiar to them. As a result, following Winograd and Flores (1986), and Herstad and Holone's (2012) ideas of building upon participants' pre-understanding and familiarity with this type of robots, a semi-autonomous omnidirectional iRobot Roombavi, vacuum cleaner, was installed in the home of some of the elderly. This activity was part of the MECS pilot study, with the purpose of developing an understanding about situated elderliness, i.e. understanding the elderly in their everyday life (Brandt et al., 2010; Light et al., 2016). In a study about ageing well from Light et al., (2015), the authors acknowledge that: "participants had little interest in tools designed specifically for 'the aged'. Instead they wanted to use the same technologies that 'everyone else' used" (p. 299). In the case of this in-motion technology, the device comes also with an app, that allows the user to control it remotely. Besides the same type of proxemics discussed in feedback between feedback between $\mathrm{H}-\mathrm{sT}$, one may control it remotely via an app, and getting a mediated feedback through it, while still being or not in relatively close area to the robot. In this way, the gestating of feedback is expanded, feedback becoming here distributed or dispersed, being given through multiple channels. In addition, the motion in itself can be discussed as a type of feedback.

\section{FINAL REMARKS AND FUTURE WORK}

In this contribution, a discussion towards the notion of feedback in relation to proxemics is being opened up. However, the paper limits the discussions around co-presence. Feedback is being conceptualized here from a quadratic anthropocentric perspective: $\mathrm{H}-\mathrm{H}, \mathrm{H}-\mathrm{nT}, \mathrm{H}-\mathrm{sT}, \mathrm{H}$ $\mathrm{mT}$. The latter two are exemplified through smartphone and a semi-autonomous vacuum cleaner, Roomba, respectively. Insights are being brought on direct feedback, feedback from an 
extended co-presence, dispersed feedback, and motion as feedback.

Finally, according to International Federation of Robotics, by 2019 there will be more than 31 million household robots devices in use. Robots are also predicted to be used highly in healthcare, or for enabling elderly to live in their home milieu longer (Bedaf et al., 2014; Kachouie et al., 2014). This encourages the ideas presented here to be further explored, with the purpose of improving the design of feedback in robots and its relation to proxemics, for the stay-at-home-elderly. Other focus areas that could be interesting to investigate in collaboration with Kampen Omsorg Pluss and other organizations are: exploration of feedback in regard to inclusion, by looking for instance to universal designed technologies; the temporality aspect of feedback, in relation to proxemics; the role of familiarity for feedback in situated elderliness for learning, when dealing with this new type of technologies in the home milieu.

\section{ACKNOWLEDGEMENTS}

This work was part of the MECS project funded by the Norwegian Research Council IKTPluss Program (Grant agreement no: 247697). We would like to thank our project funders, partners, especially to Kampen Omsorg Pluss, and to students involved in this project.

\section{REFERENCES}

Akshita, A.S., H., Indurkhya, B., Lee, E., Bae, Y. (2015) Towards Multimodal Affective Feedback: Interaction Between Visual and Haptic Modalities. In: Proceedings of the 33rd Annual ACM Conference on Human Factors in Computing Systems, CHI '15. ACM, New York, USA, 20432052.

Ashby, W.R., (1957) An Introduction to Cybernetics. Chapman \& Hall LTD, London, U.K.

Åström, K.J., Murray, R.M., (2008). Feedback Systems - An Introduction for Scientists and Engineers. Princeton University Press, Oxford, U.K.

Baecker, R.M. (1995). Readings in human-computer interaction: toward the year 2000, 2nd ed. Morgan Kaufmann Publishers, San Francisco, Calif.

Barreto, M., K., E., Nunes, N. (2013). Why Don'T Families Get Along with Eco-feedback Technologies?: A Longitudinal Inquiry. In: Proceedings of the Biannual Conference of the Italian Chapter of SIGCHI, CHItaly '13. ACM, New York, NY, USA, 16:1-16:4.

Bedaf, S., Gelderblom, G.J., de Witte, L. (2014) Overview and Categorization of Robots
Supporting Independent Living of Elderly People: What Activities Do They Support and How Far Have They Developed. Assist. Technol. 27, 88100.

Biswas, P., Langdon, P., (2012). Developing Multimodal Adaptation Algorithm for Mobility Impaired Users by Evaluating Their Hand Strength. Int. J. Human-Computer Interact. 28, 576-596.

Brandt, E., Binder, T., Malmborg, L., Sokoler, T., (2010) Communities of Everyday Practice and Situated Elderliness as an Approach to Co-design for Senior Interaction, in: Proceedings of the $22^{\text {nd }}$ Conference of the Computer-Human Interaction Special Interest Group of Australia on ComputerHuman Interaction, OZCHI '10. ACM, New York, NY, USA, 400-403.

Chittaro, L., Sioni, R., (2014). Affective computing vs. affective placebo: Study of a biofeedbackcontrolled game for relaxation training. Int. $\mathrm{J}$. Hum.-Comput. Stud., Designing for emotional wellbeing 72, 663-673.

Froehlich, J., Findlater, L., Landay, J. (2010). The Design of Eco-feedback Technology. In: Proceedings of the SIGCHI Conference on Human Factors in Computing Systems, CHI '10. ACM, New York, USA, 1999-2008.

Hall, E.T. (1966). The Hidden Dimension, 1st ed. Doubleday \& Co, New York, NY, USA.

Hall, E.T., Birdwhistell, R.L., Bock, B., Bohannan, P., Diebold, Durbin, M., Edmonson, M.S., Fischer, J.L., Hymes, D., Kimball, S.T., La Barre, W., McClellan, J.E., Marshall, D.S., Milner, G.B., Sarles, H.B., Trager, G.L., Vayda, A.P. (1968) Proxemics [and Comments and Replies]. Curr. Anthropol. 9, 83-108.

Herstad, J., Holone, H. (2012). Making Sense of Cocreative Tangibles Through the Concept of Familiarity. In: Proceedings of the 7th Nordic Conference on Human-Computer Interaction: Making Sense Through Design, NordiCHI '12. ACM, New York, NY, USA, 89-98.

Houwelingen, V., H, J., Raaij, V., Fred, W. (1989). The Effect of Goal-Setting and Daily Electronic Feedback on In-Home Energy Use. J. Consum. Res. 16, 98-105.

Kächele, M., Schels, M., Meudt, S., Kessler, V., Glodek, M., Thiam, P., Tschechne, S., Palm, G., Schwenker, F. (2014). On Annotation and Evaluation of Multi-modal Corpora in Affective Human-Computer Interaction, in: Multimodal Analyses Enabling Artificial Agents. In: HumanMachine Interaction, Springer, Cham, 35-44.

Kachouie, R., Sedighadeli, S., Khosla, R., Chu, M.T., (2014). Socially Assistive Robots in Elderly Care: A Mixed-Method Systematic Literature 
Review. Int. J. Human-Computer Interact. 30, 369-393.

Lewis, C., Norman, D.A. (1995) Human-computer Interaction, in: Baecker, R.M., Grudin, J., Buxton, W.A.S., Greenberg, S. (Eds.), Morgan Kaufmann Publishers Inc., San Francisco, CA, USA, 686697.

Light, A., Leong, T.W., Robertson, T. (2015) Ageing Well with CSCW, in: ECSCW 2015: Proceedings of the 14th European Conference on Computer Supported Cooperative Work, 19-23 September 2015, Oslo, Norway. Springer, Cham, 295-304.

Light, A., Pedell, S., Roberston, T., Waycott, J., Bell, J., Durick, J., Leong, T.W., (2016). What's special about aging. ACM Interact. 66-69.

Meurer, J., Lawo, D., Janßen, L., Wulf, V. (2016). Designing Mobility Eco-Feedback for Elderly Users. In: Proceedings of the $2016 \mathrm{CHI}$ Conference Extended Abstracts on Human Factors in Computing Systems, CHI EA '16. ACM, New York, NY, USA, 921-926.

Norman, D.A., (2013). The design of everyday things, Revised and expanded edition. ed. Basic Books, New York, NY, USA.

Pérez-Quiñones, M.A., Sibert, J.L., (1996) A Collaborative Model of Feedback in Humancomputer Interaction. In: Proceedings of the SIGCHI Conference on Human Factors in ComputingSystems, CHI '96. ACM, New York, NY USA, 316-323.

Pfeiffer, M., Schneegass, S., Alt, F., Rohs, M., (2014). Let Me Grab This: A Comparison of EMS and Vibration for Haptic Feedback in Free-hand Interaction. In: Proceedings of the 5th Augmented Human International Conference, $A H$ '14. ACM, New York, NY, USA, 48:1-48:8.

Picard, R.W. (1995) Affective Computing in M.I.T Media Laboratory Perceptual Computing Section, 321 MIT Media Laboratory, Perceptual Computing, Cambridge, MA 02139.

Piccolo, L.S.G., Baranauskas, C., Azevedo, R. (2016) A socially inspired energy feedback technology: challenges in a developing scenario. Al Soc. 1-17.

Polanyi, M., (1966). The Tacit Dimension. University of Oklahoma.

\footnotetext{
i MECS project, Institute of Informatics, University of Oslo, http://www.mn.uio.no/ifi/english/research/projects/mecs/. Retrieved: 20170512

ii International Federation of Robotics (IFR) 2017, https://ifr.org/news/31-million-robots-helping-in-householdsworldwide-by-2019. Retrieved: 20170512
}

Rhiu, I., Kwon, S., Bahn, S., Yun, M.H., Yu, W., (2015) Research Issues in Smart Vehicles and Elderly Drivers: A Literature Review. Int. J. Human-Computer Interact. 31, 635-666.

Rukavina, S., Gruss, S., Hoffmann, H., Tan, J.-W., Walter, S., Traue, H.C. (2016) Affective Computing and the Impact of Gender and Age, PLoS One 11 (3)

Sáenz-de-Urturi, Z., Zapirain, B.G., Zorrilla, A.M. (2015) Elderly user experience to improve a Kinect-based game playability. Behav. Inf. Technol. 34, 1040-1051.

Schels, M., Kächele, M., Glodek, M., Hrabal, D., Walter, S., Schwenker, F. (2014) Using unlabeled data to improve classification of emotional states in human computer interaction. J. Multimodal User Interfaces 8, 5-16.

Schwartz, T., Denef, S., Stevens, G., Ramirez, L., Wulf, V., (2013) Cultivating Energy Literacy: Results from a Longitudinal Living Lab Study of a Home Energy Management System. In: Proceedings of the SIGCHI Conference on Human Factors in Computing Systems, CHI '13. ACM, New York, NY, USA, 1193-1202.

Schwartz, T., Stevens, G., Jakobi, T., Denef, S., Ramirez, L., Wulf, V., Randall, D. (2015). What People Do with Consumption Feedback: A LongTerm Living Lab Study of a Home Energy Management System. Interact. Comput. 27, 551576.

Shneiderman, B. (1986) Designing the User Interface: Strategies for Effective Humancomputer Interaction. Addison-Wesley Longman Publishing Co., Inc., Boston, MA, USA.

Sokolova, M.V., Fernández-Caballero, A. (2015). A Review on the Role of Color and Light. In: Affective Computing. Appl. Sci. 5, 275-293.

Turner, P., (2008). Towards an account of intuitiveness. Behav. Inf. Technol. 27, 475-482.

Winograd, T., Flores, F. (1986) Understanding Computers and Cognition: A New Foundation for Design. Intellect Books.

Yeo, H.-S., Lee, B.-G., Lim, H., (2015) Hand tracking and gesture recognition system for humancomputer interaction using low-cost hardware. Multimed. Tools Appl. 74, 2687-2715.

\footnotetext{
iii Sony AIBO, 2017. Sony Aibo | The History of the Robotic Dog

Available at: http://www.sony-aibo.com/, Retrieved: 20170512

iv NAO Robot, https://www. ald.softbankrobotics.com/en/cool-

robots/nao, Retrieved: 20170512

v TurtleBot, http://www.turtlebot.com/. Retrieved: 20170512

vi iRobot Roomba, https://www.irobot.com/For-the-

Home/Vacuuming/Roomba.aspx. Retrieved: 20170512
} 